\title{
Visual imagery lowers sensitivity to hue-varying, but not to luminance-varying, visual stimuli
}

\author{
ADAM REEVES \\ Institut für Arbeitsphysiologie an der Universität Dortmund, D-4600 Dortmund 1, West Germany
}

\begin{abstract}
Visual sensitivity $\left(d^{\prime}\right)$ is reduced if a subject is instructed to maintain a visual image while the target is presented (the Perky effect; Segal, 1971). This effect, typically .6 d' units in magnitude for a colored target presented briefly on a uniform white background, is abolished if both target and background are white. (This was found with simple and complex visual images, and with FC and YN signal-detection methods.) It is concluded that visual imagery may lower the sensitivity of a psychophysical chromatic channel, but not that of an achromatic channel.
\end{abstract}

A visual target that is presented briefly on a uniform visual field may become less detectable if the subject is instructed to maintain a visual image during presentation (Segal, 1971, reviewed several studies of this effect). This reduction in sensitivity (d'), called the "Perky effect" after its discoverer (Perky, 1910), is essentially specific to each sensory modality (Segal \& Fusella, 1970), but, in the visual case, cannot be accounted for by peripheral factors such as changes in pupil diameter or eye position that might be associated with imagery (Reeves \& Segal, 1973). Reeves (1980), using a backward masking paradigm, argued that the Perky effect occurs quite early in central stages of visual processing. In this study, stimulus chromaticity and image complexity were concurrently varied, to discover which of these, if either, influences the Perky effect. If the effect occurs only in early stages of visual processing, sensory variables such as chromaticity could influence the effect, but more cognitive variables such as image complexity should not. These general expectations were tested with two signal detection methodologies, yes-no (YN) and forced-choice (FC), as detailed below.

\section{Stimulus Chromaticity}

The visual targets used by Segal and her co-workers (Segal, 1971) varied in both hue and luminance from the background on which they were presented. However, when targets differed from the background only in luminance, Reeves (1980) found, in his NM condition, that the Perky effect was greatly reduced (to about $.1 \mathrm{~d}^{\prime}$ units). In that study, complex pat-

This research was conducted while the author was at the City College of the City University of New York, and was supported by the Research Foundation of CUNY, Grant 1384. Acknowledgments are due to Jane Goldberg for technical assistance and to Dr. Sydney Segal for help and support. The author's complete address is: Institut für Arbeitsphysiologie an der Universität Dortmund, D-4600 Dortmund 1, West Germany. terned dot targets were used, precluding direct comparison with prior results. Here, the same spatially simple targets were presented in conditions in which they differed from the background either in hue and luminance, or in luminance alone, to provide a direct test of the importance of stimulus chromaticity for the Perky effect.

\section{Imagery Complexity}

Segal (1971) demonstrated the Perky effect with such rather complex images as elephants and trees. Instructions to maintain such images may have generated a cognitive load which might be responsible for the Perky effect independently of the purely visual aspects of the image, such as its experienced color, shape, and location. If so, the Perky effect ought to disappear if the image is simple enough to produce no such cognitive load. Here, instructions to image complex objects (from a list used by Segal) were compared with instructions to image the target. Because the target was simple and repeatedly presented, the load involved in creating, maintaining, and recalling an image of the target should be minimal. On the hypothesis that cognitive variables such as cognitive load do not account for the Perky effect, the Perky effect should be the same in magnitude whether images are complex or simple (Reeves \& Segal, 1973).

\section{Signal Detection Methods}

Segal (1971) used a yes-no procedure in which the signal (the target) was presented on half the trials and the subject reported its presence or absence. Such a procedure is vulnerable to criterion shifts, and, unless signal and noise distributions have equal variances, such shifts may result in spurious changes in $\mathrm{d}^{\prime}$ found from tables based on the equal-variance assumption. In fact, slopes of ROC curves obtained by the method of confidence judgments show that the ratio of signal variance to noise variance is as high as two to one 
(Segal \& Fusella, 1970). Because constancy of this ratio across experiments has not been demonstrated, and to avoid the necessity of measuring entire ROC curves, a version of the forced-choice method was also used in the present research. In this method, subjects had to report whether one or the other of two equally likely targets (a triangle and a square) had been presented. As long as subjects show no consistent bias to one target, they have a criterion $(\beta)$ of 1.0 , which places them on the negative diagonal of the ROC diagram and ensures that the calculated value of $d^{\prime}$ is then independent of the ratio of signal variance to noise variance. Both forced-choice (FC) and yes-no (YN) methods were used to provide a comparison with the earlier work on the Perky effect.

\section{METHOD}

\section{Subjects}

Thirteen randomly selected City College of New York undergraduates between 17 and 22 years of age served as subjects for $4 \mathrm{~h}$ each. They were paid independently of results. All were inexperienced in psychophysical experiments. All had normal acuity, or acuity corrected to normal, and had normal color vision as determined by a Pseudo-Isochromatic Plates test. A 14th potential subject was rejected from the experiment because she reported being unable to maintain a visual image (of a bureau) for $2 \mathrm{sec}$; the others reported that they could do so.

\begin{abstract}
Apparatus
Subjects used both eyes to look into the open end of a translucent plastic cylinder, which had its other end closed by a circular screen of the same material. The resulting visual field approximated a Ganzfeld, except that the joint between the screen and the cylinder was visible and subtended $23 \mathrm{deg}$ at the eye. The cylinder was $38 \mathrm{~cm}$ long and $15.2 \mathrm{~cm}$ in diameter. A tiny, centrally positioned black dot was positioned on the screen to aid central fixation. The targets were back-projected onto the center of the screen by a slide projector, the tungsten bulb of which could be attenuated by a Variac stabilized voltage controller. Targets, a triangle and a square, subtended $5.8 \mathrm{deg}$ and were projected for $1.5 \mathrm{sec}$. Target hue was either white (tungsten) or red (a Wratten No. 25 gelatin filter, with dominant wavelength of $621 \mathrm{~nm}$ and purity of $97 \%$ under tungsten illumination). The screen and cylinder were illuminated without visible inhomogeneities by neonwhite lamps. The luminance of the screen, measured with a MacBeth illuminator positioned at the eye, was $27 \mathrm{~cd} / \mathrm{m}^{2}$.
\end{abstract}

\section{Imagery Instructions}

The three imagery instructions were: to image an object, such as a tree, a lion, an elephant, a watermelon, or the sea; to image the target; and to image nothing. In the image-object condition, the object was read out from a list and the subject was told to form a visual image of that object. In the image-target condition, the subject was first shown the target at full intensity and was told to form an image of it, of the same shape and color but slightly larger in area. In both image conditions, the subject was told to make sure that the image was clear before the trial began, that it was held throughout the trial, and that it was "projected" onto the center of the screen. All the subjects reported that they understood this instruction and could obey it, although such an ability is not universal (Richardson, 1969). The subjects were told to report if their images were impossible to form, too small, or lost during the trial; and all such trials were replaced during the experiment. In no-imagery, the subjects were told to "empty their minds of any imagery," and to report if an image did occur dur- ing a trial; such trials were also replaced. Subjects found these instructions easy to follow, and no more than $4 \%$ of any subject's trials had to be replaced.

\section{Procedure}

Each trial consisted of the following sequence. The experimenter requested an image in the image-object condition. The subject fixated the central dot and initiated the trial when ready; then, after $1 \mathrm{sec}$, the target was presented for $1.5 \mathrm{sec}$. In the forcedchoice (FC) method, the target was either the square or the triangle, with equal probability, and the subject stated which had been presented. In the yes-no (YN) method, the target was presented on half the trials, and the subject indicated whether or not it had been presented. In YN, the target was the square for three subjects and the triangle for four. In $\mathrm{FC}$, the subjects imaged the same target they were shown in YN. Feedback of response correctedness was given after each trial.

In practice trials, the subjects gained experience with the three imagery tasks. They then received 100 no-image trials, in which target luminance was slowly reduced until performance in FC stabilized at about $80 \%$ correct. Targets were presented at this luminance for the remainder of the experiment. In experimental trials, the subjects were first shown the target at full intensity. They were then given 9 unrecorded trials, and then 25 recorded (experimental) trials. The subjects rested for $2 \mathrm{~min}$ after every block of 25 trials, and for $10 \mathrm{~min}$ after every 150 trials. Although the subjects were given feedback on each trial, they were not told how well they had performed on each block of trials or in each condition.

\section{Design}

Imagery tasks were changed every block of 25 trials, according to a different random order, for each subject, that was balanced over each set of 150 trials. Half the subjects were given FC trials for the first half of the experiment, and YN trials for the last half; the other subjects were given their trials in the reverse order. The subjects were given 200 trials in each condition of imagery in both FC and YN. Every subject was given all three imagery tasks, with both $\mathrm{FC}$ and $\mathrm{YN}$, to facilitate within-subject comparisons of the expected Perky effects. Target hue was varied between subjects: seven subjects received the white target on a white background and six subjects received the red target on the white background.

\section{RESULTS}

\section{Sensitivity}

Mean sensitivity in $d^{\prime}$ is plotted in Figure 1 against the imagery task. A drop in $d^{\prime}$ from the no-image condition (on the left) to either of the image conditions is evidence for a Perky effect. Mean d' shows such a drop when the target was red, for both YN and $\mathrm{FC}$ methods ( $\bullet$ and $\mathrm{O}$ ). When the target was white, however, the Perky effect was absent for YN ( $\square)$ and very slight for FC ( $\square$ ). These results show that the Perky effect can be abolished with targets that vary only in luminance from their backgrounds, and is the main finding of the study.

Mean sensitivity was about the same in the two imagery conditions, by both $\mathrm{YN}$ and FC methods, and suggests that the complexity of the imagery task is not a factor in producing the Perky effect.

To confirm the tendencies shown in Figure 1, the three imagery tasks were compared by a one-way ANOVA in each of the four conditions formed by 


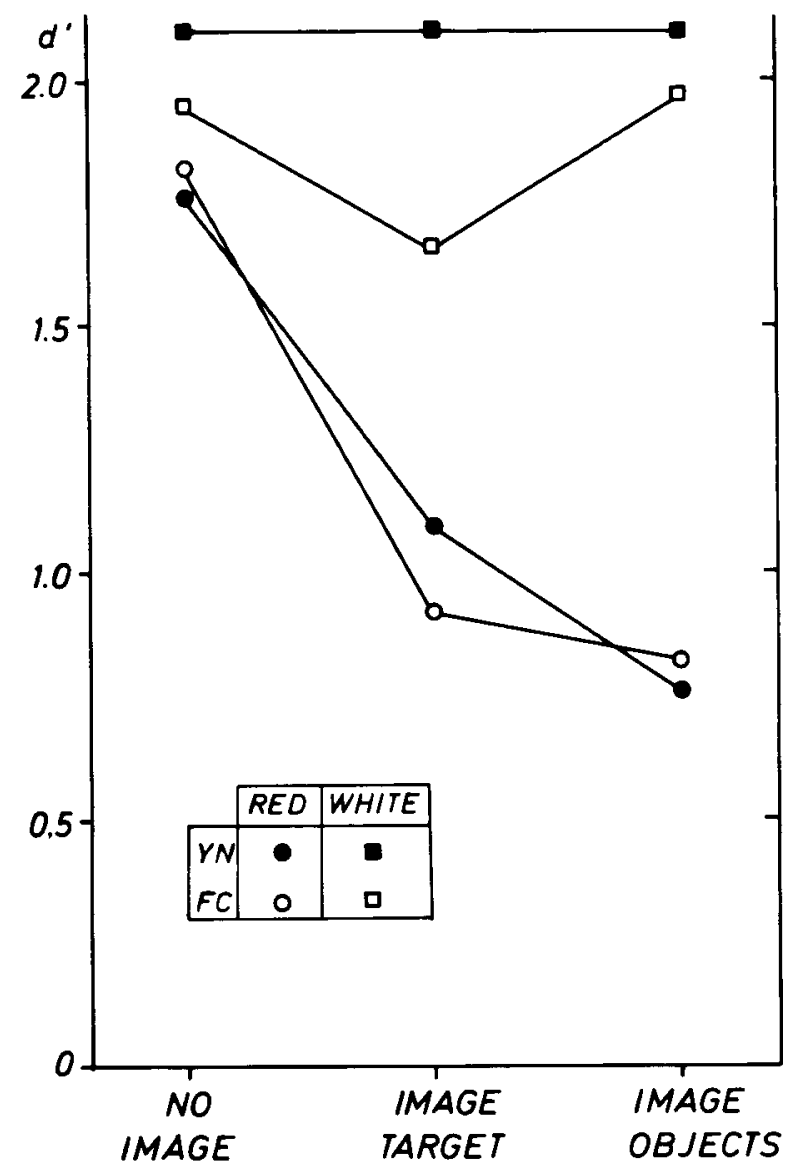

Figure 1. Sensitivity (d') as a function of imagery instruction: image nothing (no image), image target, and image objects. Background hue was neon-white. Target hue was white (circles) or red (Wratten No. 25, squares). Method was yes-no (filled symbols) or forced-choice (open symbols).

crossing method (YN or FC) by target hue (white or red). The three imagery tasks were not significantly different for the white target $(F=1.1$ in $Y N$ and $\mathrm{F}=.6$ in $\mathrm{FC} ; \mathrm{df}=2,15)$. They were different with the red target $(F=3.55$ in $\mathrm{YN}$ and $\mathrm{F}=9.86$ in $\mathrm{FC}$, $\mathrm{df}=2,18)$. As might be expected from Figure 1, with the red target the $d^{\prime}$ in image-objects was significantly different from the $d^{\prime}$ in image-nothing (planned comparisons $t=2.54$ in $\mathrm{YN}$ and $\mathrm{t}=4.33$ in $F C, d f=6$ ). The $d^{\prime}$ in image-target was also significantly different from that in image-nothing (planned comparisons $t=3.81$ in $\mathrm{YN}$ and $\mathrm{t}=3.01$ in $\mathrm{FC}$, $\mathrm{df}=6$ ). The $\mathrm{d}^{\prime}$ values in the two imagery conditions did not differ significantly $(\mathrm{t}=0.38$ in $\mathrm{YN}$ and $\mathrm{t}=$ 1.32 in FC).

\section{Bias}

The $d^{\prime}$ values were computed from the formula $d^{\prime}=z\left(P_{1}\right)-z\left(P_{2}\right)$, where $z$ is the normal transform and $P_{1}$ and $P_{2}$ are the hit and false-alarm rates in $Y N$ and the proportions of trials on which the square was reported as a square $\left(P_{1}\right)$ and as a triangle $\left(P_{2}\right)$ in $F C$. The formula is correct only if subjects are operating on the negative diagonal of the ROC curve when ROC curves have slopes other than 1.0. Because the mean value of the criterion, $\beta$, was 1.08 in $\mathrm{YN}$, the formula is probably justified. Subjects showed little bias in FC (the mean chance of reporting square was $53 \%$ ), so the formula also holds in that condition. To be quite sure, $d^{\prime}$ values were recalculated with the conservative assumption that the signal variance to noise variance ratio was 2.0 ; the recalculated mean $\mathrm{d}^{\prime}$ values were all within .15 of the values shown in Figure 1 (Reeves, 1980, gives the method for recalculating $\mathrm{d}^{\prime}$ this way).

\section{DISCUSSION}

The results obtained with the red test and white background confirm earlier findings (Segal, 1971): visual sensitivity may be considerably reduced if a subject maintains a visual image during target presentation. In Segal's research, the "Perky effect" (reduction in $\mathrm{d}^{\prime}$ due to imagery) was typically $.6 \mathrm{~d}$ ' units, close to the average of .85 found here with the red target. The Perky effect was found with both signaldetection methods and both complex and simple imagery, which extends previous work in which only complex imagery and the YN method were used (Segal, 1971).

The new finding is that the Perky effect may be abolished if the target differs only in luminance (not in hue) from its background. To replicate this new finding, an abbreviated procedure was run on five further subjects, all college students inexperienced in visual experiments. Each received 100 trials in the FC procedure with the white target. Mean $\mathrm{d}^{\prime}$ was 1.20 in no-imagery and 1.31 in image-target; the difference was not significant $(t=1.20$, $\mathrm{df}=4)$. This confirmatory result also shows that the lack of a Perky effect in Figure 1 with white targets was not an artifact of a ceiling at high $\mathrm{d}^{\prime}$ values.

Could imagery have interfered with central fixation, and thus accounted for the Perky effect with the red target? Reeves and Segal (1973) monitored eye position and found that subjects could fixate as accurately in a central 1-deg area when they maintained an image as in no-imagery; and Reeves (1980) found a Perky effect (in the BM condition) when target duration was too short for eye movements to occur. Since whatever eye movements might have occurred should be independent of target hue, it does not seem possible to account for present findingsa Perky effect with a red target but not with a whiteon the basis of eye movements. Moreover, although eye position was not monitored in this research, subjects reported they could maintain both central fixation and their visual image without difficulty during each trial. 
It seems likely that the white target was detected by psychophysical "achromatic" channels, while the red target was detected by psychophysical "chromatic" channels (Ingling \& Drum, 1973), in the conditions of this experiment. The white target provided a luminance increment on the white field, and should be detectable only by achromatic channels. The red target, when presented on a white background, should be detected by chromatic channels, since its wavelength was longer than $600 \mathrm{~nm}$ (the figure for the large, long-duration target in King-Smith \& Carden, 1976; see also Kranda \& King-Smith, 1979). If this is so, then the present research suggests that visual imagery interferes with the processing of chromatic, but not achromatic, information. (A complete test of this hypothesis would require evaluation of other targetbackground hue combinations.) That visual imagery may at least influence chromatic processing is supported by recent experiments which show McCullough effects for imaginary gratings (Finke \& Schmidt, 1978). Some support for the assumption that chromatic processes are involved is provided by the subjects' reports that the red target appeared red even when very close to threshold; the white target near threshold, of course, appeared white.

A physiological basis for an effect of imagery on chromatic processing is partly provided by Ingling and Drum (1973), who identified the psychophysically defined achromatic and chromatic channels with the phasic and tonic retinal ganglion cells of Gouras (1968), respectively. (The details of this are complex: see Ingling, 1978.) Segal and Fusella (1969) had found that the Perky effect was of the same magnitude with slowly ramped targets as it was with temporally abrupt targets. This latter result, and the present finding that the Perky effect is not obtained with white targets on a white background, is consistent with the hypothesis that imagery interferes (cortically) with information provided by tonic neural pathways alone.

\section{REFERENCES}

Finke, R. A., \& Schmidt, M. J. The quantitative measure of pattern representation in images using orientation-specific color aftereffects. Perception \& Psychophysics, 1978, 23, 515-520.

Gouras, P. Identification of cone mechanism in monkey ganglion cells. Journal of Physiology (London), 1968, 199, 533-547.

INGL!NG, C. R. Luminance and opponent color contributions to visual detection and to temporal and spatial integration: Comment. Journal of the Optical Society of America, 1978, 68, 1143-1146.

Ingl.1Ng, C. R., \& Drum, B. Retinal receptive fields:Correlations between psychophysics and electrophysiology. Vision Research, 1973, 13, 1151-1163.

King-Smith, P. E., \& Carden, D. Luminance and opponentcolor contributions to visual detection and adaptation and to temporal and spatial integration. Journal of the Optical Society of America, 1976, 66, 709-717.

KrandA, K., \& King-Smith, P. E. Detection of colored stimuli by independent linear systems. Vision Research, 1979, 19, 733-745.

Perky, C. W. An experimental study of imagination. American Journal of Psychology, 1910, 21, 422-452.

Reeves, A. Visual imagery in backward masking. Perception \& Psychophysics, 1980, 28, 118-124.

ReEves, A., \& Segal, S. Effects of visual imagery on visual sensitivity and pupil diameter. Perceptual and Motor Skills, 1973, 36, 1091-1098.

Richardson, A. Mental imagery. New York: Springer, 1969. SEgAl, S. J. Imagery: Current cognitive approaches. New York: Academic Press, 1971.

Segal, S. J., \& Fusella, V. Effects of imaging on signal-to-noise ratio with varying signal conditions. British Journal of Psychology, 1969, 60, 459-464.

Segal, S. J., \& Fusella, V. Influence of imaged pictures and sounds on detection of auditory and visual signals. Journal of Experimental Psychology, 1970, 83, 458-464.

(Received for publication June 30, 1980; revision accepted February 12, 1981.) 